\title{
La Experiencia del Campus Virtual y la Evaluación del Uso que realizan los Estudiantes de Psicologia de la Red de Internet.
}

\section{The Experience of a Virtual Campus: Evaluation of Psychology Students' Use of Internet}

\author{
Luis Ahumada Figueroa ${ }^{1}$
}

\begin{abstract}
Resumen
En este trabajo, se presenta la incorporación de un recurso de aprendizaje virtual a la asignatura de Psicología Social disponible en la página del Campus Virtual de la Universidad Católica de Valparaíso- Chile.

El sitio, dirigido a estudiantes de segundo año que cursan la asignatura mencionada, incorporó áreas temáticas no abordadas en los cursos regulares y una metodología de trabajo que favoreciera el desarrollo de una Comunidad de Aprendizaje integrando distintas perspectivas disciplinarias en el abordaje de problemas psicosociales. Específicamente, en el marco de un Aula Virtual complementaria a las clases presenciales, se desarrollaron una serie de recursos de comunicación tales como foros de discusión, votaciones respecto a problemáticas vistas en clase y chat de conversación. Se incorporaron también una serie de recursos propios de la asignatura tales como textos de apoyo y material electrónico propios del curso así como recursos de información útiles para la búsqueda de información en Psicología (PsycFirst, PsycINFO_1887, Econlit, entre otros).

Al comienzo y al final del curso (anual), se evaluó el impacto de esta metodología en el uso que realizan los estudiantes de la red de Internet, aplicando una encuesta elaborada específicamente para evaluar la utilización de la red de Internet por parte de los estudiantes.

Mediante esta metodología de enseñanza aprendizaje se logró que el estudiante incorporara información adicional a los temas vistos en Cátedra, además se observó un cambio significativo en la evaluación respecto a la finalidad del uso de la Red y su utilización como recurso de aprendizaje.

La utilización de esta herramienta virtual y esta nueva metodología de enseñanzaaprendizaje, mejora la calidad de la docencia, no tanto en el aprendizaje de los contenidos propios de la Psicología Social, como en el desarrollo de competencias transversales relacionadas principalmente con la gestión de la información y el conocimiento en el contexto de las Tecnologías de la Información y la Comunicación (TICs).
\end{abstract}

1 Académico, Escuela de Psicología, Pontificia Universidad Católica de Valparaíso. email: lahumada@ucv.cl 
Palabras Claves: Uso de Internet, Metodologías de Enseñanza y Aprendizaje, Tecnologías de la Información y las Comunicaciones, Aprendizaje Virtual.

\begin{abstract}
This paper discusses the integration of a virtual learning procedure into a Social Psychology course. This resource is available through the Virtual Campus at the Pontificia Universidad Católica de Valparaíso, Chile (www.ucv-cv.cl).

The site, designed for second year psychology students enrolled in the course, included topics not covered in the regular course and a methodology that fosters the development of a learning community.

Content and methods were sought to integrate various disciplinary perspectives addressing psychosocial problems. More specifically, the virtual classroom complemented regular classroom instruction by providing a number of communication resources such as: discussion forums, opinion polls of controversial topics covered in class, and a chat room. Additionally, it included support texts, electronic materials, as well as instructions to search psychology-related databasis (i.e., PsychFirst, PsychINFO, and Econlit).

Through a survey designed to assess the students' use of Internet, pre (at the beginning of the academic year) and post (at the end of the year-long course) measures were obtained to examine the impact of this virtual learning procedure. Results show that students searched for information not covered in class and there was a significant change in the results assessed on the use of Internet as a learning procedure.

The use of a teaching-learning methodology that includes a virtual tool improves the quality of teaching, by developing life long learning skills related to information management and knowledge of information and communication technologies (TICs).
\end{abstract}

Key words: Internet use, Teaching and learning methods, Information and Communication Technologies, Virtual Learning.

\section{Introducción}

\section{Contexto Actual de las Organizaciones Universitarias Chilenas}

El sistema de educación superior chileno ha sufrido cambios importantes en los últimos veinte años. Cambios que se han traducido en una creciente diversificación del conjunto de instituciones $y$, por ende, de carreras y programas de formación (Ministerio de Educación de Chile, 1999).

La diversidad de instituciones y carreras universitarias ha tenido como principal conse- cuencia una creciente preocupación en torno a la calidad de la formación (UNESCO, 1998). La pregunta central que se ha planteado es: ¿que se entiende por calidad de la formación y cuáles son las competencias necesarias con las que deben contar los profesionales universitarios?

El constante cambio del entorno, impone a las organizaciones universitarias requerimientos de información y de acción con el fin de lograr una mejor adaptación (Davenport, y Prusak, 2001; Levine, 2002, Teare, Davies y Sandelands, 2002). Las fluctuaciones en la economía, las innovaciones tecnológicas, las reformas legales, la mayor especialización de la 
fuerza de trabajo, la flexibilización laboral y el aplanamiento de la estructura organizacional, son factores que se citan con frecuencia como ejemplos de los cambios en el entorno (Sisto, 2001; Barkema, Baum, y Mannix, 2002).

En este contexto, se ha resaltado la importancia de la gestión del conocimiento para poder adaptarse a los requerimientos del entorno. De hecho, para diversos autores (Karnoe, 1996; Nonaka, Takeuchi, y Umemoto, 1996; Tsoukas y Chia, 2002), los conocimientos que posee una organización son su principal ventaja competitiva.

Como toda organización, las Universidades tienen su propia forma de crear, adquirir, interpretar, transformar, difundir, recuperar y utilizar su conocimiento. Desde una perspectiva socio-constructivista (Ahumada, 2002; Ascorra, 2002; Jonson et al., 2002), los miembros de una comunidad universitaria, incorporan no sólo los contenidos aprendidos formalmente sino los procesos que hacen posible la incorporación de dichos contenidos.

La Universidad debe aprovechar sus recursos competitivos. Debe hacer un uso intensivo de sus conocimientos con el fin de mejorar la formación de sus estudiantes y sus fortalezas como organización (Herrera, 1998; Lindauer, 1998). Estos procesos se inscriben en lo que se denomina la gestión del conocimiento organizacional (Brockmann y Anthony, 2002, Fiol, 2002; Hatch y Schultz, 2002).

Vignolo (1998) señala la gestión del conocimiento fomenta a la organización a obtener penetración (conocimiento sobre su medio externo) y entender su propia experiencia (conocimiento sobre su medio interno). En otras palabras, a generar y utilizar, espacios de interacción humanas, que permitan desarrollar los activos intangibles que apoyan a la organización en el logro de sus objetivos (Mamaghani, 2002).

En cuanto a la formación universitaria, se menciona la necesidad de contar con un modelo de enseñanza-aprendizaje cuyo én- fasis no esté puesto tanto en los contenidos aprendidos cuanto en el proceso de creación de dichos contenidos y en los recursos que hacen posible y necesario el desarrollo de nuevos conocimientos. Se señala la necesidad de potenciar redes de aprendizaje (Harasim et al., 2000 Teare, Davies y Sandelands, 2002), que permitan a los estudiantes aprender de forma conjunta, en el lugar, el momento y al ritmo que les resulte más apropiado (Perry-Smith y Shalley, 2003; Duart y Sangrà, 2000).

Las investigaciones que han intentado dilucidar las variables más importantes que dicen relación con la calidad de la docencia universitaria, han encontrado que los estudiantes ponen el énfasis en que el docente esté disponible para ellos y en compartir vivencias personales; mientras que los profesores ponen énfasis en la necesidad de que los alumnos discutan conceptos claves con sus compañeros, se fijen metas y analicen resultados (Zúñiga, 1998). Como se puede apreciar, la mirada sobre el proceso docente aparece sobre aspectos distintos aunque complementarios; unos enfatizan la calidad de la docencia desde el punto de vista de la apertura del profesor mientras que otros lo hacen desde el punto de vista de los métodos conducentes a la obtención de determinados resultados.

Para Montecinos (2003), una docencia de calidad debe estimular la relación entre el profesor y el alumno, estimular la colaboración entre los profesores como miembros de una comunidad profesional de aprendizaje, favorecer el aprendizaje activo y colectivo, integrar la teoría con la práctica situada en la escuela, la formación continua, la incorporación de los conocimientos provenientes de las investigaciones combinando teoría y práctica, respetar las diferentes capacidades y estilos de aprendizaje utilizando múltiples estrategias.

Los estudiantes universitarios, para tener éxito como alumnos y posteriormente como profesionales, necesitan además conocer herramientas computacionales y ser hábiles en 
su utilización. Los resultados obtenidos por López-Mena (2001) al analizar el uso de la tecnología en estudiantes y profesionales de la Psicología, muestran una ausencia del uso de la tecnología de las comunicaciones y de la red de Internet tanto en los procesos formativos como en el ejercicio profesional.

Sin embargo, las competencias que casi todos los trabajadores necesitan en el trabajo, según Vergara y Isaac (1988), incluyen habilidades para:

1. adquirir y evaluar información

2. organizar y mantener información

\section{3. interpretar y comunicar información}

4. usar computadores para procesar información

Desde un enfoque de competencias se denomina "analfabetismo funcional" a aquellas personas que no manejan habilidades computacionales mínimas. En la sociedad del conocimiento, lo relevante es lo que hacemos con la información, como logramos integrarla en unidades significativas y aplicarla en nuestro contexto cotidiano. El concepto de "empowerment" sintetiza este cambio de enfoque (Robbins, Crino y Fredendall, 2002)

Es decir, necesitamos pasar de una era de la información a una era del conocimiento. Necesitamos un modelo de enseñanza-aprendizaje cuyo énfasis no esté puesto tanto en los contenidos aprendidos cuanto en el proceso de creación de dichos contenidos y en los recursos que hacen posible y necesario el desarrollo de los conocimientos (Moreno, 1998).

A juicio de diversos autores (Zurita, 1998; Zúñiga, 1998; Pérez, 2000), un modelo de aprendizaje basado en los recursos requiere un cambio de paradigma para los educadores. Nuevos y variados recursos de aprendizaje son una parte central del proceso de aprendizaje, no sólo un apoyo a la enseñanza tradicional. En este modelo, los estudiantes debieran ser capaces de planificar la bús- queda de información, localizar, recuperar, procesar, registrar, presentar y evaluar la información para poder así aplicarla y contrastar su validez empírica.

En este nuevo modelo o enfoque, el profesor se convierte en un guía del aprendizaje facilitándose al estudiante los medios y recursos para ir descubriendo y construyendo su propio conocimiento. Herrera (1998), pone énfasis en la transformación del proceso de enseñanza-aprendizaje desde uno centrado en el docente a otro que esté centrado en el aprendiz y transformar nuestro paradigma de la "comunidad académica" en otro definido como "comunidades de aprendices".

El contexto actual de las Universidades chilenas y el desarrollo futuro de la formación del alumnado, plantean un nuevo desafío que se materializa en el programa denominado Campus Virtual impulsado por la Pontificia Universidad Católica de Chile. Este programa se propone impulsar innovaciones metodológicas que faciliten el desarrollo de habilidades y competencias requeridas en la sociedad del conocimiento, tales como, expresión oral y escrita, destrezas computacionales, capacidad de aprender por cuenta propia, creatividad, conocimiento y comprensión del mundo y capacidad para asumir tareas de conducción y de trabajo en equipo.

\section{La Experiencia del Campus Virtual}

En atención al desarrollo de nuevas Tecnologías de Información y a nuevos modelos pedagógicos basados en la interacción y la retroalimentación, el proyecto del Campus Virtual surge a mediados del año 2000 como una iniciativa de la Universidad tendiente tanto a impartir cursos no presenciales como a apoyar la docencia presencial de las distintas carreras y programas de estudio que se imparten en la Universidad.

El modelo educativo del Campus Virtual está basado en un sistema educacional cooperativo en que interactúan Profesores, Alum- 
nos y Tutores, usando de apoyo a Internet y a las Tecnologías de la Información y las Comunicaciones (TIC). Su objetivo, según queda explicitado en el proyecto, es permitir la adquisición de contenidos particulares y la elaboración de conocimientos nuevos, a partir del perfeccionamiento de habilidades por parte de los estudiantes. Se pretende propiciar el desarrollo del proceso de aprendizaje; esto es, la integración del nuevo conocimiento y de la estrategia utilizada para aprenderlo.

Esta nueva comprensión de la educación se mueve desde estar centrada en el profesor a centrarse en el alumno, en modificar el rol del educador desde la entrega de contenidos, hasta convertirse en un mediador efectivo y significativo, que sea el soporte fundamental para que el alumno sea capaz de elaborar contenidos auténticos.

Entre las herramientas que se pueden utilizar y con las que cuenta el Campus Virtual se encuentran los siguientes sitios de ingreso:

\section{Espacios y Herramientas Propias del Aula}

Unidades de Trabajo: espacio donde el alumno obtiene el material de estudio del curso.

- Documentos: en esta sección el alumno encontrará todo el material del curso (presentaciones, lecturas, papers, etc.).

- Glosario: en esta sección podrán encontrarse las definiciones de algunos términos y conceptos que serán tratados en la asignatura y que pueden estar acompañados de documentos y/o de sitios web.

- Recursos del aula: en esta sección podrán encontrarse materiales complementarios al curso, tanto documentos digitales como direcciones web.

- Actividades: en esta sección se encontrarán las actividades (guías de trabajo, tareas, etc.) del curso.
- Evaluaciones: en esta sección se encontrarán las evaluaciones del curso (pautas de corrección, pruebas anteriores, etc.).

Recursos de Comunicación: son aquellas herramientas destinadas a favorecer la interacción tanto del profesor con sus alumnos como entre los propios alumnos. El objetivo es fomentar la construcción del conocimiento de los alumnos a través de estos espacios de interacción.

- Foro: el objetivo del foro es fomentar la discusión y colaboración de los participantes, por lo que es esencial intentar responder y complementar mensajes de compañeros o profesores, en vez de sólo agregar nuevos mensajes independientes.

- Votaciones: esta herramienta permite conocer la opinión de todos los alumnos del curso respecto de algún tema en particular que será expuesto por el profesor según las necesidades del curso o de los mismos alumnos.

- Archivos colaborativos: esta herramienta ha sido diseñada pensando en el trabajo grupal que puedan desarrollar los alumnos sin estar reunidos físicamente en un mismo lugar (5 ó 6 alumnos). Un alumno del grupo o el profesor, crean el primer archivo y el resto podrá ir realizando los aportes que estimen conveniente actualizando la versión ya existente.

- Chat: es un espacio de comunicación abierto cuya característica principal es la posibilidad de conversar en tiempo real o en línea temas de interés común sin pauta de conversación previa.

\section{EsPaCiOS Y HERRAMIENTAS DE LA Plataforma Común a Todas las Aulas}

Recursos metodológicos: corresponden a herramientas que facilitan el uso y manejo de información, tanto en recopilación y comprensión, como en la transmisión y organización de ésta. Los recursos incluidos en esta 
opción tienen como objetivo prestar un apoyo al aprendizaje de los participantes, en relación con técnicas de estudio.

Biblioteca de recursos: es una serie de recursos que pueden servir de referencia, como herramientas en la preparación de trabajos o actividades de sus cursos, así como para uso de interés personal, se ofrecen recursos de información útiles para la búsqueda de información en Psicología (PsycFirst, PsycINFO_1887, Econlit, entre otros). La Biblioteca contiene recursos de variados temas y son accesibles, al igual que en el caso de los Recursos Metodológicos, para todos los usuarios del Campus Virtual.

Con el fin de aportar a la formación de los estudiantes de Psicología en el aprendizaje y uso de las tecnologías de la información y las comunicaciones, la cátedra de Psicología Social de la Escuela de Psicología de la PUCV, incorporó como parte de sus actividades académicas el uso intensivo de las herramientas del Campus Virtual anteriormente descritas.

A partir de una problemática social libremente escogida con relación a los contenidos propuestos en el programa del curso, los estudiantes conformados en grupos de cinco alumnos iniciaron un trabajo de investigación consistente en la construcción de un marco teórico, recogida de información empírica, análisis y comunicación de los resultados en formato de artículo científico.

Inicialmente los estudiantes utilizaron los archivos colaborativos para la elaboración de sus trabajos y, posteriormente, una vez presentados oralmente los trabajos al curso, se subían estos al Campus Virtual con un foro de discusión propuesto por el grupo. Los estudiantes podían acceder libremente a los foros que desearan (10 foros), teniendo una evaluación tanto los trabajos realizados como la consigna entregada en el foro y la participación en los mismos.

El principal objetivo de nuestra investigación fue analizar la percepción que tienen los estudiantes la cátedra de Psicología Social de la Escuela de Psicología de la PUCV acerca del uso de Internet. Específicamente, se pretendía conocer diversos tópicos relacionados con las dificultades, frecuencia y finalidad de uso de la red de Internet, la utilización de ésta como recurso de aprendizaje, los aspectos positivos y negativos de la infraestructura de computadores disponibles y el acceso a la misma. Aspectos didácticos específicos del uso de Internet en Psicologia y una valoración general respecto a la enseñanza virtual y el uso de las tecnologías de la información y las comunicaciones por parte de los alumnos.

\section{Método}

Para el logro de los objetivos antes señalados, se utilizó una metodología de carácter descriptivo cuantitativo.

1. Selección y características de la muestra. La muestra estuvo compuesta por los alumnos matriculados regularmente en el curso de Psicología Social impartido en la Escuela de Psicología de la Pontificia Universidad Católica de Valparaíso-Chile. En total la muestra esta constituida por 48 estudiantes de los cuales 18 eran varones y 30 eran mujeres (37\% y 63\% respectivamente). La media de edad fue de 19 años teniendo el $83 \%$ de la muestra entre 18 y 20 años.

2. Instrumentos. Se elaboró y validó (con estudiantes de otros cursos de Psicología) un instrumento que permitiera recoger información referida al uso que realizan los estudiantes de Internet y la percepción que tienen de esta como apoyo a su formación de Psicólogos (Anexo 1). Este cuestionario con formato tipo Likert (siendo 1 totalmente en desacuerdo y 7 totalmente de acuerdo), está dividido en ocho apartados que recogen información referente a:

- evaluación de la finalidad del uso de la red

- evaluación de la frecuencia del uso de la red 
- experiencia previa de utilización de Internet

- evaluación de Internet como recurso de aprendizaje

- percepción del sistema web

- equipos e infraestructura

- aspectos didácticos de la Internet en Psicología.

- valoración general de Internet

3. Procedimiento de Recogida de información. El instrumento anteriormente descrito fue aplicado a la muestra seleccionada al comienzo y al final del curso de Psicología Social (Curso Anual). En el cuestionario se pedía como datos de identificación la edad, sexo y rol de estudiante.

4. Análisis de la información. Para el análisis de la información se utilizó el programa SPSS 10.5 realizándose con este programa todos los análisis estadísticos correspondientes.

\section{Resultados}

A continuación se presentan los principales resultados obtenidos tras la aplicación del cuestionario en cada una de las dimensiones señaladas al finalizar el curso. Se entrega la puntuación media obtenida en los respectivos ítems del cuestionario y los porcentajes correspondientes; las puntuaciones van de 1 (Totalmente en desacuerdo) a 7 (Totalmente de acuerdo); siendo la puntuación 4 (Indeciso).

Evaluación de la Finalidad del Uso de la Red

$\mathrm{Al}$ analizar la finalidad de uso de la red podemos señalar que los alumnos de Psicología utilizan la red principalmente para leer el correo electrónico (media de 5,5, estando el $47 \%$ totalmente de acuerdo y el $25 \%$ bastante de acuerdo con esta afirmación) y para realizar los trabajos de los cursos (media de 4,4 , estando el $17 \%$ bastante de acuerdo y
$30 \%$ algo de acuerdo). Otros usos que se señalan son el establecer vínculos y relaciones (media de 3,9), leer los periódicos y estar actualizado (media de 3,4); siendo la puntuación más baja para aprender mejor los contenidos de los cursos (media de 2,8) y para el acceso a la Biblioteca Virtual de la Universidad (media de 1,6).

De los resultados encontrados respecto a la finalidad de uso de la red, sorprende lo poco que se utiliza Internet para acceder a la Biblioteca Virtual de la Universidad (61\% totalmente en desacuerdo), lo que se relaciona con el poco material bibliográfico y artículos de revistas que se bajan de Internet. Por otra parte, el establecer vínculos y relaciones así como leer los periódicos y estar actualizado, son los ítems que presentan mayor dispersión dentro de esta dimensión (1.84 y 1.90 de desviación típica, respectivamente).

\section{Evaluación de la Frecuencia del Uso de la Red}

$\mathrm{Al}$ analizar el número de días que los estudiantes utilizan Internet a la semana, estos señalan que lo hacen de dos a tres días a la semana. Con un promedio de $45^{\prime \prime}$ minutos al día. Se debe tener presente que un $40 \%$ señala utilizar Internet sólo un día a la semana, un 12\% dos días a la semana y un $20 \%$ tres días a la semana, por lo que la frecuencia de utilización de Internet se puede considerar como baja. En cuanto al tiempo de conexión a Internet, $29 \%$ señala conectarse entre 30 " y $45^{\prime \prime}$ y un $27 \%$ entre $45^{\prime \prime}$ y $60^{\prime \prime}$ con un promedio de $45^{\prime \prime}$ minutos al día; lo que demuestra que si bien la frecuencia de conexión es baja el tiempo de conexión a Internet es relativamente alto.

\section{Experiencia de Uso}

Respecto a los principales problemas que se mencionan para la utilización de Internet, los estudiantes señalan que Internet es lenta a la hora de buscar y bajar la información necesaria (media de 4,8; $25 \%$ totalmente de acuerdo). Asimismo señalan, en orden decreciente como problema la falta de co- 
nocimiento o experiencia de trabajo con la red (media de 4,$6 ; 25 \%$ algo de acuerdo, $17 \%$ bastante de acuerdo y $21 \%$ totalmente de acuerdo), la dificultad para acotar bien la información requerida (media de 4,3; 39\% algo de acuerdo y $13 \%$ bastante de acuerdo), la falta de conocimiento de las palabras claves de búsqueda (media de 4,$2 ; 26 \%$ algo de acuerdo y $21 \%$ bastante de acuerdo) y de sitios de búsqueda (media de 4,$1 ; 30 \%$ algo de acuerdo y $19 \%$ bastante de acuerdo). La falta de conocimiento del idioma inglés (media de 3,$6 ; 52 \%$ en desacuerdo), no aparece como un problema especialmente relevante en el uso de la red.

\section{Percepción del Sistema Web}

Los estudiantes de Psicología estiman que Internet resulta fácil y segura de usar con fines académicos (media de 4,6;55\% de acuerdo). Sin embargo, no todos están de acuerdo que Internet ayude a aprender mejor los contenidos de los cursos (media de 3,8; $21 \%$ indeciso y $35 \%$ en desacuerdo) y que resulte más amena para aprender que el sistema tradicional de clases (media de 3,2; $29 \%$ indeciso y $52 \%$ en desacuerdo).

\section{Equipos e Infraestructura}

Respecto al acceso a equipos computacionales, la mayoría manifiesta que es fácil acceder a la utilización de computadores (media de 5,$2 ; 73 \%$ de acuerdo) ya sea que estén en la propia casa o en casa de amigos; estando la mayor parte de estos conectados a la Red (media de 5,0; 69\% de acuerdo). Sin embargo, no todos están de acuerdo en que sea fácil acceder a la utilización de los Computadores de la Universidad (media de 4,3; 40\% en desacuerdo).

\section{Evaluación de Internet como Recurso de Aprendizaje}

En cuanto a la evaluación de Internet como recurso de aprendizaje, los estudiantes señalan que si tuviesen apoyo a sus clases a través de Internet, la usarían constantemente (media de 5,4; 78\% de acuerdo), dado que beneficia el aprendizaje siempre y cuando se ofrezca algo novedoso casi a diario (media de 5,2; 73\% de acuerdo). Por otra parte, los estudiantes señalan que el mayor poder de Internet es la posibilidad que ofrece de interactuar con otros (conseguir cosas, hablar con otros, etc.) (media de 5,4; 79\% de acuerdo).

Respecto a los contenidos y la administración de los sitios WEB, los estudiantes señalan no estar de acuerdo en que estos son útiles sólo si son simples y sintéticos (media de 3,$4 ; 54 \%$ en desacuerdo), y que sólo se aprende en Internet en la medida en que se pueda recibir rápida retroalimentación y ayuda de quienes administran los contenidos (media de 3,$7 ; 27 \%$ indeciso y $41 \%$ en desacuerdo).

\section{Aspectos Didácticos de la Internet en Psicología}

A pesar de esta valoración positiva de Internet como recurso de aprendizaje los estudiantes señalan que pocas veces buscan contenidos de Psicología en Internet (media de 4,4; $57 \%$ de acuerdo) y menos artículos y bibliografía complementaria de lo que ven en clases (media de 3,$7 ; 12 \%$ indeciso y $45 \%$ en desacuerdo). Los estudiantes no tienen claro si los sitios WEB de Psicología son aburridos o no (media de 3,$7 ; 47 \%$ indeciso), dado que pocas veces buscan contenidos de Psicología en Internet.

\section{Valoración General de Internet}

La Figura 1 muestra la valoración general de Internet. Los estudiantes señalan que ésta es una buena herramienta de apoyo a su formación como psicóloga/o (media de 5,7;38\% algo de acuerdo, $29 \%$ bastante de acuerdo y $27 \%$ totalmente de acuerdo). Sin embargo, las opiniones están bastante dividas respecto a si se matricularían o no en un curso no presencial (media de 4,$4 ; 15 \%$ en desacuerdo, $25 \%$ indeciso y $20 \%$ totalmente de acuerdo). 


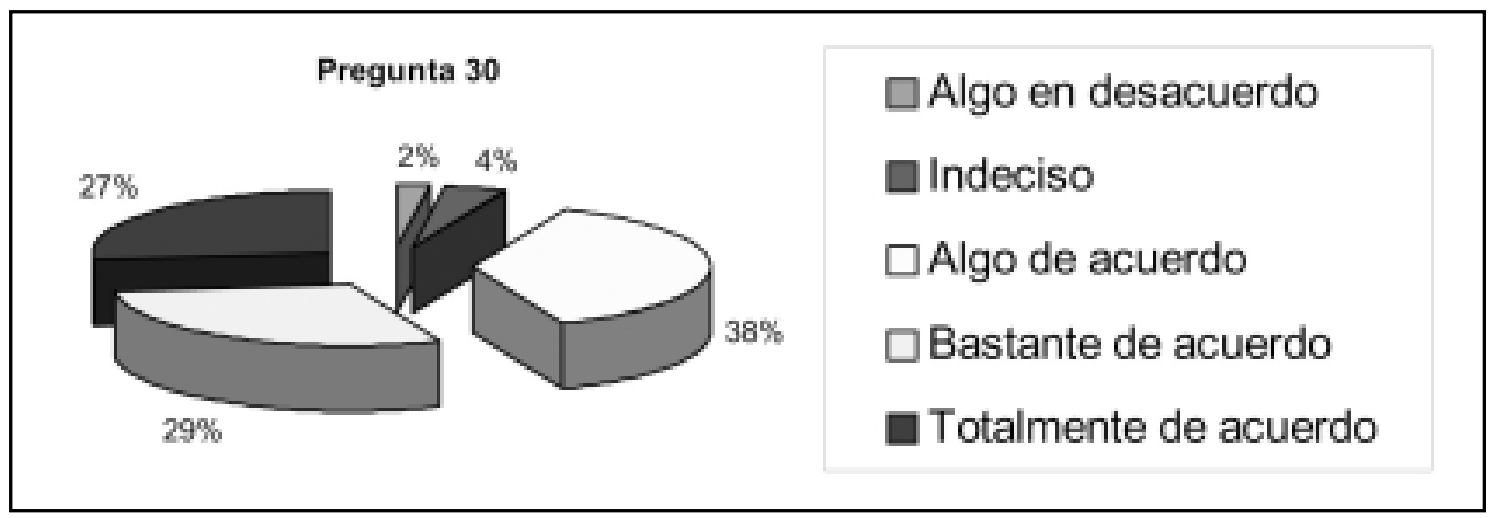

Figura 1. Valoración General de Internet

Diferencias entre Sujetos de Distinto Sexo Respecto de su Valoración en la Utilización de Internet

De acuerdo a la prueba no paramétrica $U$ de Mann-Whitney, es posible señalar que sólo existen diferencias significativas respecto de su valoración hacia la finalidad del uso de la red. Los alumnos -a diferencia de las alumnas- evalúan positivamente los fines hacia los que se destina Internet y reconocen obtener mejor provecho de ello $(\mathrm{p}=0.048)$.

En el resto de las dimensiones estudiadas (frecuencia uso, experiencia previa, recurso de aprendizaje, percepción del sistema, equipos e infraestructura, aspectos didácticos y valoración general) no existen diferencias significativas por lo que se debe ser cauteloso a la hora de sacar conclusiones respecto a la variable género. Estos resultados coinciden con los encontrados por Gutiérrez, Álvarez y Jarne (2002), quienes no encontraron diferencias significativas entre hombres y mujeres respecto al uso y valoración de Internet.

\section{Diferencias Entre la Primera y la Segunda Instancia de Aplicación de la Encuesta de Evaluación del Uso de Internet}

A través de la prueba no paramétrica de Friedman y con $p=0,05$, es posible aceptar diferencias respecto de la evaluación inicial sobre el uso de Internet y su correspondien- te evaluación de seguimiento al final del curso (anual). La opinión de los sujetos mejora con la utilización de la red de Internet por parte de los estudiantes.

Mediante esta metodología de enseñanza aprendizaje se logró que el estudiante incorporara información adicional a los temas vistos en Cátedra, se observó además un cambio significativo en la evaluación respecto a la finalidad del uso de la Red y su utilización como recurso de aprendizaje $(p<0.05)$.

\section{Discusión}

Los alumnos del curso de Psicología Social investigado utilizan la red principalmente para leer el correo electrónico y para realizar los trabajos de los cursos; sin embargo, este uso a juicio de los estudiantes no necesariamente significa aprender mejor los contenidos. Internet se utiliza poco para acceder a la Biblioteca Virtual de la Universidad, lo que se relaciona con el poco material bibliográfico y artículos de revistas que se bajan de la red.

Los estudiantes utilizan Internet dos a tres días a la semana. El tiempo de conexión a Internet, es en promedio de 45 " minutos al día; lo que demuestra que si bien la frecuencia de conexión es baja el tiempo de conexión a Internet es relativamente alto.

Respecto a los principales problemas que se mencionan para la utilización de Internet; 
en orden decreciente son: la lentitud a la hora de buscar y bajar la información necesaria, la falta de conocimiento o experiencia de trabajo con la red, la dificultad para acotar bien la información requerida, la falta de conocimiento de palabras claves y de sitios de búsqueda.

Los resultados anteriores concuerdan con los encontrados por López-Mena (2001) en donde aparece como problema principal manifestado por los estudiantes la falta de conocimiento o experiencia de trabajo con la red. Por otra parte, la falta de conocimiento del idioma inglés, no aparece como un problema especialmente relevante en el uso de la red.

Los estudiantes de Psicología estiman que Internet resulta fácil y segura de usar con fines académicos. Sin embargo, no todos están de acuerdo que Internet ayude a aprender mejor los contenidos de los cursos y que resulte más amena para aprender que el sistema tradicional de clases.

Respecto al acceso a equipos computacionales, la mayoría manifiesta que es fácil acceder a la utilización de computadores ya sea que estén en la propia casa o en casa de amigos; estando la mayor parte de estos conectados a la Red. Sin embargo, no todos están de acuerdo en que sea fácil acceder a la utilización de los computadores de la Universidad. Los resultados de esta investigación contrastan con los encontrados por López-Mena (2001) y Gutiérrez, Álvarez y Jarne (2002), en donde la dificultad de acceso aparece como uno de los principales inconvenientes.

En cuanto a la evaluación de Internet como recurso de aprendizaje, los estudiantes señalan que si tuviesen apoyo a sus clases a través de Internet, la usarían constantemente, dado que beneficia el aprendizaje siempre y cuando se ofrezca algo novedoso casi a diario. Por otra parte, los estudiantes señalan que el mayor poder de Internet es la posibilidad que ofrece de interactuar con otros. Estos resultados coinciden con los encontrados por Gutiérrez, Álvarez y Jarne (2002), en donde la interacción y la flexibilidad de uso aparecen como las principales ventajas.

Respecto a los contenidos y la administración de los sitios WEB, los estudiantes señalan estar en desacuerdo en que estos son útiles sólo si son simples y sintéticos, y que sólo se aprende en Internet en la medida en que se pueda recibir rápida retroalimentación y ayuda de quienes administran los contenidos.

A pesar de esta valoración positiva de Internet como recurso de aprendizaje los estudiantes señalan que pocas veces buscan contenidos de Psicología en Internet y menos artículos y bibliografía complementaria de lo que ven en clases. Al parecer los estudiantes no tienen claro si los sitios WEB de Psicología son aburridos o no, dado que pocas veces buscan contenidos de Psicología en Internet.

Finalmente, en cuanto a la valoración general de Internet los estudiantes señalan que ésta es una buena herramienta de apoyo a su formación como psicóloga/o. Estando bastante dividas las opiniones respecto a si se matricularían o no en un curso no presencial. Al parecer existiría un perfil de estudiante que estaría totalmente en desacuerdo con inscribirse en un curso no presencial y otro que estaría totalmente de acuerdo con tal posibilidad.

Podemos concluir pues que la utilización del Campus Virtual y esta nueva metodología de enseñanza-aprendizaje, mejora la calidad de la docencia, no tanto en el aprendizaje de los contenidos propios de la Psicología Social, como en el desarrollo de competencias transversales relacionadas principalmente con la gestión de la información y el conocimiento en el contexto de las Tecnologías de la Información y la Comunicación (TICs). El análisis de un mejor aprendizaje de los contenidos propios de la asignatura no estaba dentro de los objetivos de la investigación y la metodología empleada no permite sacar conclusiones a este respecto; sería interesante en futuras investigaciones abordar específicamente este punto, sin 
embargo, el análisis cualitativo del uso de los foros de discusión permite señalar un aumento de la capacidad crítica y de reflexión de los alumnos respecto a los principales tópicos vistos en clase.

\section{Referencias}

Ahumada, L. (2002). El aprendizaje organizacional desde una perspectiva evolutiva y constructivista de la organización. Revista de Psicología de la Universidad de Chile, XI, 1, 139-148.

Ascorra, P. (2002). Acción organizacional y socioconstruccionismo. Revista de Psicología de la Universidad de Chile, XI, 1, 165-173.

Barkema, H. BAum, J. \& Mannix, E. (2002) Management challenges in a new time. Academy of Management Journal, 45, 5, 916-930.

Brockmann, E. \& Anthony, W. (2002). Tacit knowledge and strategic decision making. Group $\mathcal{E}$ Organization Management, 27, 4, 436-455.

DAVENPORT, T. \& PRUSAK, L. (2001). Conocimiento en acción: como las organizaciones manejan lo que saben. Buenos Aires: Pearson Education.

DuART, J. y SANGRÀ, A. (2000). Aprender en la virtualidad. Serie: biblioteca de educación. Nuevas tecnologías. Barcelona, España: Gedisa.

FIOL, C. M. (2002). Capitalizing on paradox: the role of language in transforming organizational identities. Organization Science, 13, 6, 653-666.

Gutiérrez, J., Álvarez, E. y Jarne, A. (2002) Recursos didácticos basados en Internet para un curso de Psicología patológica. Anuario de Psicología, 33, 3, 433-452.

Harasim, L. et al. (2000). Redes de aprendizaje. Serie: biblioteca de educación. Nuevas tecnologías. Barcelona, España: Gedisa.
HАтсн, M. \& Schultz, M. (2002). The dynamics of organizational identity. Human Relation, 55, 8, 989-1018.

Herrera, R. (1998). Bases para el desarrollo cualitativo de la docencia universitaria. Chile: Cinda.

Herrera, R. (1998). Bases para el desarrollo cualitativo de la docencia universitaria. Chile: Cinda.

JoHnSON, S. et al. (2002) Team development and group processes of virtual learning teams. Computers E Education, 39, 379-393.

KARnOE, P. (1996). The social process of competence building. International Journal of Technology Management, Special Issue on Unlearning and Learning for Technological Innovation, 11(7/8), 770-789.

Levine, D. (2002) Thinking about doing: on learning from experience and the flight from thinking. Human Relations, 55, 10, 1251-1268.

Lindauer, B. G. (1998). Defining and measuring the library's impact on Campus wide outcomes. College $\mathcal{E}$ Research Libraries, 59(6): 546-570.

López-Mena, L. (2001) Aplicaciones de Internet en la Formación de los Estudiantes de Psicología: Estudio de Caso. Revista de Psicología de la Universidad de Chile, X, 1, 9-19.

MAMAGHANI, F. (2002) Information Technology Knowledge Sharing Using Case-Based Reasoning. Information Systems Management, 19, 4, 13-20.

Ministerio de Educación de Chile (1999). Programa de Mejoramiento de la Calidad y la Equidad de la Educación Superior (MECESUP). Santiago: MINEDUC.

Montecinos, C. (2003) Desarrollo profesional docente y aprendizaje colectivo. Revista Psicoperpectivas, II, 105-128. 
Moreno, M. et al. (1998) Conocimiento y cambio: los modelos organizadores en la construcción del conocimiento. Barcelona: Paidós, Temas de Psicología.

NonAKA, I., TAKeUChI, H. y UMEMOTO, K. (1996). A theory of organizational knowledge creation. International Journal of Technology Management, Special Issue on Unlearning and Learning for Technological Innovation, 11(7/8), 833-845.

Pérez, A. (2000). Bibliotecas y centros de documentación en un entorno virtual. En Duart, J. y Sangrà, A. (2000) Aprender en la virtualidad. Serie: biblioteca de educación. Nuevas tecnologías. Barcelona, España: Gedisa.

Perry-Smith, J. y Shalley, C. (2003) The social side of creativity: a static and dynamic social network perspective. Academy of Management Review, 28, 1, 89-106.

Robbins, T. CRino, M. \& Fredendall, L. (2002) An integrative model of the empowerment process. Human Resource Management Review. 12, 419-443.

SISTO, V. (2001). Flexibilidad, Cultura y Complejidad, aportaciones desde una perspectiva polifónica al análisis organizacional. Ponencia presentada en el XXVIII Congreso Interamericano de Psicología. $1^{\circ}$ de Agosto de 2001; Santiago de Chile.
Teare, R. Davies, D. \& SAndelands, E. (2002). Organizaciones que aprenden y formación virtual. Serie: biblioteca de educación. Nuevas tecnologías. Barcelona, España: Gedisa.

Tsoukas, H. y ChiA, R. (2002). On organizational becoming: rethinking organizational change. Organization Science, 13, 5, 567-582.

UNESCO. (1998). Conferencia mundial sobre educación superior en el siglo XXI. Visión y acción. París, Unesco.

Vergara, A. y IsAac, J. (1998). Tecnología de la comunicación e informática como apoyo a la docencia. Santiago, Chile: Cinda.

VignOlo, C. (1998). Los Determinantes de la Innovación en empresas manufactureras chilenas. Estudios Públicos, 70, Santiago Chile.

ZúÑIGA, M. (1998). Algunos criterios para la formulación de una estrategia integral de docencia en educación superior: una mirada desde la relación enseñanza aprendizaje. Santiago-Chile: Cinda.

Zurita, R. (1998). Crisis de identidad y de misión de la universidad: la formación profesional. Santiago-Chile: Cinda. 


\section{Anexo 1 \\ Cuestionario de Utilización de la Red de Internet}

Evaluación de la Finalidad del Uso de la Red

1. Internet la utilizo principalmente para aprender mejor los contenidos de los cursos

2. Internet la utilizo principalmente para realizar los trabajos de los cursos

3. Internet la utilizo principalmente para leer mi correo electrónico

4. Internet la utilizo principalmente para acceder a la Biblioteca Virtual de la UCV

5. Internet la utilizo principalmente para leer los periódicos y estar actualizado

6. Internet la utilizo principalmente para establecer vínculos y relaciones

Evaluación de la Frecuencia del Uso de la Red

7. Señale el número de días que utiliza Internet a la semana:

8. Señale la cantidad promedio de minutos que utiliza Internet al día:

\section{Experiencia Previa de Utilizacion de Internet}

Mi experiencia previa de utilización de Internet me indica que los principales problemas radican en:

9. La falta de conocimiento o experiencia de trabajo con la red

10. La falta de conocimiento del idioma ingles

11. La falta de conocimiento de las palabras claves de búsqueda

12. La falta de conocimiento de los sitios de búsqueda

13. La dificultad para acotar bien la información requerida

14. Internet es lenta a la hora de buscar y bajar la información necesaria

\section{Percepcion del Sistema Web}

15. Internet me ayuda para aprender mejor los contenidos de los cursos

16. La utilización de Internet me resulta más amena para aprender que el sistema tradicional de clases

17. Internet resulta fácil y segura de usar con fines académicos

\section{Equipos e Infraestructura}

18. Me es fácil acceder a la utilización de Computadores (Casa, amigos, etc.)

19. Me es fácil acceder a la utilización de Computadores conectados a la Red

20. Me es fácil acceder a la utilización de los Computadores de la Universidad

Evaluación de Internet como Recurso de Aprendizaje

21. Los contenidos de Internet son útiles sólo si son simples y sintéticos

22. Sólo se aprende en Internet en la medida en que se pueda recibir rápida retroalimentación y ayuda de quienes administran los contenidos.

23. Si tuviese apoyo a mis clases a través de Internet, la usaría constantemente.

24. Creo que Internet beneficia el aprendizaje si ofrece algo novedoso casi a diario.

25. El mayor poder de Internet es su interactividad (conseguir cosas, hablar con otros, etc.).

Aspectos Didácticos de la Internet en Psicología

26. Los sitios WEB de Psicología son aburridos.

27. Pocas veces busco contenidos de Psicología en Internet.

28. En Internet busco ejemplos o casos reales de lo que veo en clases de Psicología.

29. En Internet busco artículos y bibliografía complementaria de lo que veo en clases.

Valoracion General de Internet

30. Me parece que Internet es una buena herramienta de apoyo a mi formación como psicóloga/o.

31. De ser posible, en el futuro me matricularía en un curso no presencial 
